\title{
Dynamics of Birth Interval Components in Manipur
}

\author{
Md. Abdul Latif and N. Sharat Singh* \\ Department of Statistics, Thoubal College under Manipur University, Imphal
}

\begin{abstract}
The birth interval is one of the major determinants of high fertility populations. Under cluster sampling scheme, a cross sectional as well as community based study of 1193 eligible women was carried out using a pre-tested and semi-structural interview schedule during May to November, 2012 in the four valley districts of Manipur, a border Indian State with Myanmar to investigate pattern of birth interval components. Cox' regression model $\left[h(t, Z)=h_{O}(t) \Psi(Z)\right]$ explores infant mortality, lactation, use of contraceptive devices, son preference and religion are found significant $(P<0.01)$ factors on the dynamics of birth interval components in the present censored data.
\end{abstract}

Key Words: censored data, relative risk, lactation, infant mortality, son preference

\section{Introduction}

Human fertility solely depends on the duration of birth intervals (Bongaarts and Potter 1983). This interval, especially closed birth interval, is composed of three major components namely postpartum amenorrhoea (PPA), waiting time to conception and gestation. The last one, gestation that is the pregnancy period is treated to be constant while PPA, the time interval between the termination of pregnancy and the first successive ovulation, is a physiological process which varies in a complex fashion (Lantz et al. 1992; Nath et al. 1993; Clegg 2001; Awang 2003, Singh et al., 2012). The second component - waiting time to conception, the time interval between the resumption of menses after a pregnancy until the beginning of the next pregnancy, is highly influenced by socio-economic, demographic, cultural, and behavioural factors (Kathleen et al. 1989; Lantz et al. 1992; Rao et al. 2006; Singh et al. 2007, Singh et al. 2011).

In most developing countries almost all women practice breastfeeding their children initially. Breast milk not only provides immunological protection against common childhood disease such as allergies, diarrhoea, obesity and certain metabolic and others (PIP, 2003). Each woman experiences a period of temporary infecundibility following childbirth commonly referred to as the post partum non-susceptible period during which she does not ovulate. The practice of intensive and lengthy breastfeeding is associated with prolonged post partum amenorrhoea in societies where it is nearly universal (Srinivasan et al., 1989; Mukherjee et al., 1994; Dissanyake, 2000). Bongaarts and Potter (1983) have demonstrated that in populations without access to modern form of contraception, birth intervals are determined by the duration of breastfeeding. In consensus statement, a group of international scientists agreed that breastfeeding provides more than $98 \%$ protection from pregnancy during the first six months post partum period if the mother is fully or nearly full breastfeeding and has not experienced vaginal bleeding after the fifty sixth day post partum (Kennedy et al., 1989). The duration of post partum amenorrhoea is quite long especially in rural areas and therefore it is one of the responsible factors for the longer birth interval among Indian women when compared to Western women (Srinivasan et al., 1989; Nath et al., 1993; Dissanyake, 2000). Most of their studies have clearly indicated a direct link between duration of breastfeeding and post partum amenorrhoea. But apart from breastfeeding, many researchers have shown that several socio-demographic factors affect the duration of post partum amenorrhoea (Nath et al., 1993; Mukherjee et al., 1994; Singh, 2007, Aryal, 2006, Singh et al., 2012).

\section{Objective}

To manage the high fertility in India, the present study is initiated to investigate empirically the differential pattern of duration of birth interval components say 'post partum amenorrhoea' and 'waiting time to conception' with respect to various socio-demographic factors of interest.

\section{Materials and methods}

A cross sectional as well as community based study was conducted in the four valley districts of Manipur - Bishnupur, Imphal East, Imphal West, and Thoubal. It is one of the north eastern Indian states having international borders with Myanmar. Using a pre-tested and semi-structural interview schedule, the survey was completed during the period from May to November, 2012 with the reference date of $1^{\text {st }}$ May 2012 with cluster sampling technique. It comprises of 1193 eligible women. The study subjects are considered into account after excluding the ones who did not meet the two criteria viz., (i) she must have had at least two pregnancies in her 
life time and (ii) her most recent pregnancy must have resulted in two live births. As the present study is confined in censored data and also to explore the causal factors, a survival analysis technique particularly Cox' regression analysis is carried out through SPSS. The results are expressed in terms of P-value of the Wald test of the regression coefficients ( $\beta$ ), relative risk (RR) of the explanatory variables with $95 \%$ confidence interval (CI).

\section{Cox' regression model}

The simple form of the model is $h(t, Z)=h_{O}(t) \Psi(Z)$ where $h_{O}(t)$ is the baseline failure rate or typical hazard and $\Psi(\mathrm{Z})$ a parametric link function bringing in the covariates. It satisfies $\Psi(0)=1$ and $\Psi$ $(Z) \geq 0$ for all $Z$. The commonly used form of $\Psi$ is $\Psi(Z)=Y(Z, \beta)=\exp \left(\beta^{\prime}, Z\right)$ known as the 'log linear form'. Thus for the individual with covariate vector $Z$, the hazard function $h(t, Z)$ can be represented as $h(t, Z)$ $=h_{0}(t) \exp \left(\underline{\beta}^{\prime}, Z\right)$ so that the ratio, $h(t, Z) / h_{0}(t)=\exp \left(\beta^{\prime}, Z\right)$ represents the relative risk of failure. Further, $\log \left[h(t, Z) / h_{0}(t)\right]=\left(\beta^{\prime}, Z\right)$ is the usual form of a linear regression model and hence the name 'log linear model'. Since the regression coefficients are constants and covariates are fixed, then the hazards $h(t, Z)$ and $h_{o}(t)$ are proportional and hence the name Proportional Hazards. Interpreting the model involves examining the coefficients for each explanatory variable. For continuous variables, the parameter $\beta$ denotes the effect of a unit change in the independent covariates on the 'log' of the hazard rate, after adjustment the other variables. For categorical covariates, $\beta$ represents the deviation of a specified group from the hazard of the reference group. The exponential of the coefficients, ' $\exp (\beta)$ ' called relative risk (RR) also use to express the hazard of a specified group as a proportion of the baseline hazard.

\section{Response variables}

The response variables - the components of birth intervals namely the duration of PPA and waiting time to conception are taken subject to the last births only to control data recall error. Since ovulation itself is difficult to identify, the reliable estimate of the end of amenorrhoea is the return of menstruation itself. The duration of PPA was estimated as an interval between the termination of conception and the return of first menses. Women reporting the termination of amenorrhoea before the survey date were considered uncensored cases, and their duration of PPA was the interval between the termination of conception and the return of the first menses. On the other hand, women reporting continuance of amenorrhoea even after the survey date were considered censored cases, and their duration variable was the interval between the termination of conception and survey date. An eligible woman who conceives before the survey date is considered to be uncensored in case of estimating the duration of waiting time to conception. The duration variable is quantified by the time interval between end of PPA and date of conception. The time interval observed from the woman who does not conceive till the date of survey is considered to be censored case and the duration is the time interval between the end of PPA and the survey date.

\section{Variable specification}

The response variables are post partum amenorrhoea (PPA) and waiting time to conception (in month). Explanatory variables of interest are age at menarche (AGME), age at marriage of husband (AGMH) and wife (AGMW), age at delivery (AGDV), parity (PETY), infant mortality - death of previous child in infancy (DPCL: dead $=1$, alive $=0$ ), number of living son $(\mathrm{NLSN})$, number of living daughter (NLDR), duration of marriage (DUMR, in year), couple's desire number of son (CDNS), sex of the previous child (SPCL: male $=1$, female $=0$ ), type of feeding (TYPF: completely breast fed $=1$, otherwise $=0$ ), lactation (LACT, duration of breast feeding in month), use of contraceptive device (UCDV, effectively used $=1$, otherwise $=0$ ), educational level (completed years in education) of husband (EDUH) and wife (EDUW), religious differential due to Hindu (RELH: Hindu=1, Others $=0$ ) and Islam (RELI: Islam $=1$, others $=0$ and family monthly income (FINC: in ' 000 rupee).

\section{Analysis and Results}

After adjustment of the effects of other explanatory variables only three - duration of breast feeding $(\mathrm{P}<0.01)$, infant mortality $(\mathrm{P}<0.01)$ and family income $(\mathrm{P}<0.05)$ are found to have their significant impacts on the variation of PPA (Table-1). The stepwise method of the Cox regression is again employed to investigate the most influential factors contributing higher risk of being short duration of PPA and the results are also manifested in Table -2. Five variables - infant mortality, family income, lactation, type of feeding and Islamic religion are identified to be such determinants in the last fitted model that is in $5^{\text {th }}$ step. In the first step, lactation 
(LACT) has a very high impact on the dynamics of PPA $(\mathrm{P}<0.01)$ in such a way that it has $12 \%$ reduced risk of being short duration of PPA when the mothers breast feeding practice six month (RR=0.98 with 95\% CI: 0.98 $0.99)$. Next to LACT, infant mortality becomes to be high risk factor of PPA in $2^{\text {nd }}$ step ( $P<0.01, R R=2.13$ with 95\%CI: 1.44-3.14). In this way, FINC $(\mathrm{P}<0.01)$, RELI $(\mathrm{P}<0.05)$ and TYFD $(\mathrm{P}<0.05)$ are gradually found to be high risk indicators of PPA in addition to LACT and DPCL in the $3^{\text {rd }}, 4^{\text {th }}$ and $5^{\text {th }}$ steps. In the last fitted model, the death of previous child during infancy has a linkage with the short duration of PPA. It marks 2.14 times higher risk in shortening the duration of PPA when the previous infant is death than those of infant survive keeping constant the effects of four other variables. While controlled the effects of DPCL, LACT, FINC and RELI, type of feeding (TYFD) has significant impact on duration of PPA in the last model. The breastfed mothers could have longer duration of PPA, reducing risk of $18 \%$ on the hazard of the duration (RR=0.83 with 95\%CI: 0.69-0.99). Here the religious differential also exists to be significant contribution on the duration. At least 33\% more risk of shortening PPA is observed in Islam than those combination of Hindu, Meitei and Christian (RR=1.37 with 95\% CI: 1.05-1.78).

In this analysis, only five factors - DPCL $(\mathrm{P}<0.01)$, SPCL $(\mathrm{P}<0.05)$, DNSW $(\mathrm{P}<0.05)$, LACT $(\mathrm{P}<0.01)$ and EDUW $(\mathrm{P}<0.05)$ are found to be significant factors influencing the duration of waiting time to conception when adjusted the effects of other variables (Table-1). The death of previous child in infancy throws a highly significant contribution in reducing waiting time to conception. The high risk 2.67 times (95\%CI: 1.66-4.30) would be faced the women whose previous child is death compared to alive. Son preference also highly influences waiting time to conception. The couple's desire number of has more contribution son $(\mathrm{P}<0.05)$ of being short waiting time. It is worthwhile to mention again that lactation (LACT) has lesser hazards thereof in the sense that the increase in lactation lengthens the duration of waiting time to conception $(\mathrm{P}<0.01)$. In the last model of stepwise method, the six factors have been identified to have risk in the dynamics of waiting time to conception. They are DPCL $(\mathrm{P}<0.01)$, SPCL $(\mathrm{P}<0.01)$, CDSN $(\mathrm{P}<0.05)$, UCDV $(\mathrm{P}<0.05)$, LACT $(\mathrm{P}<0.01)$ and RELH $(\mathrm{P}<0.01)$ manifested in Table-2. In the first step, lactation has the highest impact on the duration. Next to lactation, death of previous child during infancy is identified to be the most influential factor. In this way, religion, sex of previous child, desire number of son by wife and use of contraceptive device are gradually confirmed to be high risk factors of birth interval dynamics in step 3, 4, 5 and 6 respectively. Lactation has $21 \%$ reduced risk of being short birth interval in each six months increasing of duration of breastfeeding ( $R R=0.97$ with 95\% CI, 0.96-0.97). If the previous child is died during infancy, 2.51 times more risk is exhibited than those of child alive at least one year. In crude sense, it has $150 \%$ more hazard of shortening the waiting time in death of previous child than those alive during infancy ( $\mathrm{RR}=2.51$ with 95\% CI: 1.60-53.93). The religion differentials are also found to be significant contribution in the variation of waiting time. Hindu and similar category (RELH) has longer waiting time than that of others - Meiteism, Islam and Christian. It is observed that $19 \%$ more risk of short waiting time in the religious groups other than Hindu. The couple's desire number of son has direct linkage with short waiting time which is statistically significant. It contributes $29 \%$ more risk in son preference than that of daughter ( $\mathrm{RR}=1.29$ with $95 \% \mathrm{CI}$ : 1.05-1.58). The finding is again explored that the couples those who use the contraceptive device effectively can reduce significantly the duration of waiting time to conception. It can reduce $17 \%$ risk of short waiting time after adjusted the effects of five factors - LACT, DPCL, SPCL, DNSW and RELH in the last fitted model.

\section{Discussion}

The present finding again confirms the fact that lactation prolongs the duration of post partum amenorrhoea. It is thought to be caused by a physiological mechanism which originates in the hypothalamus, located in the basal region of the brain (Aguirre, 1996). The women's hypothalamic nuclei release gonadotropinreleasing hormone $(\mathrm{GnRH})$ in a regular, episodic fashion, which in turn triggers the pulsatile and episodic release of Luteinizing hormone (LH) and follicle stimulating hormone (FSL) from the pituitary. This biological process is also known as the hypothalamic pulse generator (HPG) (Mc Neilly et al., 1985). The releases of certain amounts of LH have been found to be crucial for normal ovarian activity and fecund menstrual cycles (Mc Neilly et al., 1985). It is also observed that normal patterns of GnRH and LH release can be disrupted by external factors, such as heavy physiological exercise or activity, or psychological stress, in addition to breastfeeding (Loucks, 1990; Genazzani et al., 1991). The exact neurotransmitter signals, which interrupt the release of GnRH and LH during these activities, remain to be completely elucidated. In the case of breastfeeding women during the post partum period, however, the suckling stimulus may be the primary signal which disrupts the normal pattern of hormonal activity (Tay et al., 1993). This general mechanism represents a direct link between the external environment (i.e. stressors) and the internal regulation of the reproductive system (Jones and Palloni, 1994). Thus breast feeding and ovulation are antagonistic, because either prolactin or gonadotropins can not be produced simultaneously or prolactin blocks the action of gonadotropins, and hence resulting to depress the onset of ovulation. The present finding that lactational duration certainly delay the duration of post partum amenorrhoea is also supported by a number of studies (Srinivasan et al., 1989; Nath et al., 1993; Dissanyake, 
2000; Singh, 2010; Singh et al., 2012). The death of previous child during infancy terminates earlier the duration of post partum amenorrhoea. However, it seems to have no direct role towards the regulation of post partum amenorrhoea. But it works as an intermediate variable through practice of lactation which is incorporated with the findings of Lantz et al., (1992), Dissanyake (2000) and Singh (2012).

The income level of the couple has an impact on the dynamics of post partum amenorrhoea in the present study .The shorter duration of post partum amenorrhoea is associated with high income. Perhaps, it has again a direct linkage with the educational level of the couple. In other words, education of couple is positively associated with the risk of return of menses. This may be due to the fact that if couples are more educated they would practice conditionally or unconditionally artificial/bottle feeding more frequently, which would possibly less suckling at the nipples of the breast. This view is supported by Kennedy et al., (1989) and Mukherjee et al., (1994). It is again incorporated with the finding of Kathleen et al., (1989) indicating the increase in educational level of wife is associated with short duration of post partum amenorrhoea. After controlling the effects of four factors namely lactation, death of previous child during infancy, income level and religious differentials, the type of feeding has significantly lower risk of shortening the duration of post partum amenorrhoea. Irrespective of all factors, religious differential causes a high significant effect $(\mathrm{P}<0.01)$ on regulation of post partum amenorrhoea in the study population. It is still found significant $(\mathrm{P}<0.05)$ in Islam women when adjusted the effects of four factors say death of previous child during infancy, lactation, income and type of feeding. Islam women have a higher risk of shorter post partum amenorrhoea than that of Hindu and Christian counterparts. The possible reason may be the mother's nutritional status. Generally Islam couples are in the category of low income group resulting into poor nutrition.

Irrespective of all covariates, the couples those who marry late have a tendency towards quick child bearing and try to compensate their earlier lost reproductive period in order to have a desire number of children. Consequent upon their effective reproductive period is being short and hence directly results into short waiting time to conception. The view is incorporated with the findings of Clegg (2001), Singh et al., (2007), Singh et al., (2010). On the contrary, when the effects of various covariates are adjusted, the impact of age at marriage of both spouses is statistically insignificant in the study. This is thought to be caused by the educational levels of couple in the sense that the age at marriage is significantly associated with educational level achieved.

Perhaps, death of previous child during infancy limits the duration of waiting time to conception through emotional and psychological feeling of the couples. Even irrespective of lactation, an infant death may exert a psychological pressure on the parents to make up the lost as early as practicable. The behaviour of child replacement effect involves a deliberate decision by the couples to compensate the death child leading to short waiting time to conception. On the other hand, presence of an infant or young child and strain of rearing the child may reduce the desire sexual relations which may result in reducing coital frequency and hence leading to longer waiting time to conception. This view is supported by the findings of Lindstrom et at., (2000), Youssef, (2006), Singh et al., (2007), Singh et al., (2011) etc. The death of previous child in infancy has a highly significant impact on the waiting time to conception. Indeed, couples have preference to male offspring not only in the study population but in many other societies in the world too since boys are preferred for long term benefits such as family fortune and perpetuation of family name. Consequently sex preference has a strong bearing on waiting time to conception. Couples prefer to go for number of children in order to achieve the desire number of sons. Possibly, whenever there is a female child and if the couple intend to go for more births it exerts a psychological and emotional pressure to have the next child of the desired sex quickly and hence leads to shorter duration of waiting time to conception. The lactation has a high significant impact on the regulation of waiting time to conception. In one hand, it might be thought to be caused by the fact that most breastfed mothers have lesser educational status leading to unemployed. These mothers are generally found to have offspring closely to their desire number of children so that the degree of eagerness for getting the excess number is reduced and hence the duration becomes to be significantly increased. On the other hand, employed mothers are usually educated and performed late marriage as well as practicing less breast feeding. Consequently they compensate the lost reproductive period by curtaining waiting time to conception to achieve their desire number of child.

\section{Conclusion}

The dynamics of the durations of post partum amenorrhoea and waiting time to conception play a tremendous role in fertility regulation through birth interval. Among the determinants, infant mortality, lactation and religion have significant impacts on both duration variables in the Cox's stepwise regression models. While family income and type of feeding influences the PPA, son preferences, measured by sex of previous child and couple's desire number of son, and use of effective contraceptives have also been found to be significant factors of waiting time to conception. The analysis highlights the fact that some socio-demographic factors have vivid contribution towards the regulation of components of birth intervals specifically the post partum amenorrhoea despite its dependency on the physiological process with a very complex phenomenon and waiting time time to conception. However, the present study is confined only rural valley area of Manipur. It may therefore be 
suggested that such a study may be taken up at a larger scale covering entire geographical area of the state. This will help to asses the causal effects on the variation of the major components of human birth intervals through which the state high fertility level may somewhat be checked.

\section{Acknowledgement}

The authors are indebted to University Grants Commission, India for financial support of the Research Project from which the present data is utilized with reference to its sanction letter No. F-5-463/201112(MRP/NERO)/11745 dated the $26^{\text {th }}$ December, 2011.

\section{References}

[1]. Aguirre, G.P., 1996, The determinants of post Partum amenorrhea: A multi state hazard approach. Centre for Demography and Ecology Working Paper, University of Wisconsin-Madison; 96-03.

[2]. Aryal, T. R., 2006, Retrospective reporting of the duration of post-partum amenorrhea: A survival analysis. Kathmandu University Medical Journal; 4(2): 211-217.

[3]. Awang, H., 2003, Determinant of waiting time to third pregnancy using censored linear regression. Journal of Biosocial Science, 35: 59-70.

[4]. Bongaarts, J. and Potter, R. G., 1983, Fertility, Biology and Behaviour. $1^{\text {st }}$ Edition. New York, Academic Press.

[5]. Clegg, E. J., 2001, Starting, spacing and stopping in the reproductive histories of outer Hebridean families. Journal of Biosocial Sciences; 33: 405-526.

[6]. Cox, D. R., 1972. Regression models and life tables (with discussions). Journal of Royal Statistical Society, Series B; 34 : 187-220.

[7]. Dissanyake, L., 2000. The influence of child mortality and breastfeeding on interlive birth intervals in Sri Lanka. Journal of Family Welfare; 46(1): 229-239.

[8]. Genazzani, A. R., Petraglia, F., Genazzani, A. D., Amato, G., D’Ambrogio, G., Angioni, S. and Bidzinska, B., 1991, Perspective on stress-induced amenorrhoea, Pp. 321-326. In: Stress and relative disorders from adaptation to dysfunction. Genazzi, A. R., Nippi, C., Petraglia, F. and Martignoni, E. (eds.), Carnforth, U. K, Parthenon Publishing.

[9]. Jones, R. E. and Palloni, A., 1994, Investigating the determinants of post partum amenorrhoea using a Multistage hazards model approach. Annals of the New York Academy of Science; 709: 227-230.

[10]. Kathleen, F., Sandra, L. H., Chowdhury, A. K., Becker, G., Allen, H. and Menken, J., 1989. Birth interval dynamics in Rural Bangladesh and maternal weight. Demography; 26(3): 425-437.

[11]. Kennedy, K.I., Rivera, R. and McNeilly, A.S., 1989, Consensus statement on the use of breastfeeding as a family planning method. Contraception; 39(5): 477-496.

[12]. Lantz, P., Paartin, M. and Palloni, A., 1992, Using retrospective surveys for estimating the effects of breastfeeding at child spacing on infant and child mortality. Population Studies; 46: 121-139.

[13]. Linstrom, D.P. and Berhanu, B., 2000, The effects of breast feeding and birth spacing on infant and earlychildhood mortality in Ethiopia. Social Biology; 47: 1-17.

[14]. Loucks, A. B., 1990, Effects of exercise training on the menstrual cycle: existence and mechanisms. Medicine and Science in Sports and Exercise; 22: 275-280.

[15]. McNeilly, A. S., Glasier, A. and Howie, P. W., 1985, Endocrine control of lactational infertility, pp. 1-16. In: Dobbing, J. (ed.), Maternal nutrition and lactational infertility. New York: Raven Press.

[16]. Mukherjee, S., Bhattacharya, B. N. and Singh, K. K., 1994, Effect of breastfeeding on post partum amenorrhoea in Eastern Uttar Pradesh. Janasamkhya; 12(1\&2): 107-131.

[17]. Nath, D. C., Singh, K. K., Land, K. C. and Talukdar, P. K., 1993, Breastfeeding and post partum amenorrhoea in a traditional society: A hazards model analysis. Social Biology; 40: 74-84.

[18]. Population Information Program (PIP), 2003, Birth Spacing: Three to Five saves Lives. Population Reports; 30(3): 1-24.

[19]. Rao, S. R., Townsend, J. and Askew, J., 2006, Correlates of Inter-Birth Intervals: Implications of Optimal Birth Spacing Strategies of Mozambique. Population Council Working Paper. Assessed on <www.popcouncil.org/pdfs/frontiers/FR_Final Reports/Mozam_OBSI> (Retrieved March, 2009).

[20]. Singh, N. S., Narendra, R. K. and Hemochandra, L., 2007, Determinants of waiting time to conception in Manipuri women. Kuwait Medical Journal; 39(1): 39-43.

[21]. Singh, N. S., Singh, S. N. and Narendra, R. K., 2010. Demographic and socio-economic determinants of birth interval dynamics in Manipur: A survival analysis. Journal of Health Allied Sciences; 9(4): 3.

[22]. Singh, N. S., Singh, S. N. and Narendra, R. K., 2011, Differential pattern of duration of waiting time to conception of women in Manipur. Studies in Home and Community Sciences; 4(2): 144 -154.

[23]. Singh, N. S., Singh, S. N. and Narendra, R. K., 2012, Postpartum amenorrhoea among Manipuri women: A survival analysis. Journal of Health, Population and Nutrition; 30(1): 93-98.

[24]. Srinivasan, K., Pathak. B. and Pandey, A., 1989, Determinants of breastfeeding and post partum amenorrhoea in Orissa. Journal of Biosocial Sciences; 21: 365-371.

[25]. Tay, C. K., Glasier, A. F. and McNeilly, A. S., 1993, Effects of antagonists of dopamine and opiates on the basal and GnRH-induced secretion of luteinizing hormone, follicle stimulating hormone and prolactin during lactational amenorrhoea in breastfeeding women. Human Reproduction; 8: 532.

[26]. Youssef, R. M., 2005, Duration and determinants of inter birth interval: community-based survey of women in southern Jordan. Eastern Mediterranean Health Journal; 11(4): 559-572.

Table-1: Cox' Regression analysis (Adjusted) of birth interval components with socio-demographic variables

\begin{tabular}{|l|c|c|c|c|}
\hline \multirow{2}{*}{ Variables } & \multicolumn{2}{|c|}{ PPA } & \multicolumn{2}{c|}{ Waiting time to conception } \\
\cline { 2 - 5 } & RR(with 95\%CI) & P-value & RR(with 95\%CI) & P-value \\
\hline AGME & $0.97(0.92-1.01)$ & $>0.05$ & \multicolumn{2}{c|}{ NC } \\
\hline AGMW & $1.03(0.99-1.07)$ & $>0.05$ & $1.06(0.97-1.15)$ & $>0.05$ \\
\hline AGMH & $0.99(0.97-1.01)$ & $>0.05$ & $1.01(0.99-1.03)$ & $>0.05$ \\
\hline
\end{tabular}


Dynamics of Birth Interval Components in Manipur

\begin{tabular}{|c|c|c|c|c|}
\hline PETY & $1.04(0.86-1.26)$ & $>0.05$ & $0.93(0.78-1.11)$ & $>0.05$ \\
\hline NLSN & $0.90(0.76-1.07)$ & $>0.05$ & $1.12(0.92-1.37)$ & $>0.05$ \\
\hline NLDR & $0.94(0.79-1.13)$ & $>0.05$ & $1.08(0.89-1.31)$ & $>0.05$ \\
\hline SPCL & $1.01(0.85-1.21)$ & $>0.05$ & $1.26(1.06-1.50)$ & $<0.05$ \\
\hline DPCL & $2.07(1.36-3.17)$ & $<0.01$ & $2.67(1.66-4.30)$ & $<0.01$ \\
\hline DUMR & \multicolumn{2}{|l|}{$\mathrm{NC}$} & $1.05(0.97-1.14)$ & $>0.05$ \\
\hline TYPF & $0.85(0.70-1.02)$ & $>0.05$ & $1.02(0.83-1.25)$ & $>0.05$ \\
\hline LACT & $0.98(0.97-1.00)$ & $<0.01$ & $0.97(0.96-0.97)$ & $<0.01$ \\
\hline UCDV & \multicolumn{2}{|l|}{$\mathrm{NC}$} & $1.17(0.99-1.37)$ & $>0.05$ \\
\hline RELH & $0.99(0.84-1.17)$ & $>0.05$ & $0.83(0.68-1.01)$ & $>0.05$ \\
\hline RELI & $1.34(0.99-1.82)$ & $>0.05$ & $1.12(0.82-1.52)$ & $>0.05$ \\
\hline EDUW & $0.99(0.97-1.01)$ & $>0.05$ & $1.02(1.00-1.04)$ & $<0.05$ \\
\hline EDUH & $1.01(0.99-1.04)$ & $>0.05$ & $0.99(0.97-1.02)$ & $>0.05$ \\
\hline FINC & $1.01(1.00-1.02)$ & $<0.05$ & $0.99(0.98-1.00)$ & $>0.05$ \\
\hline CDNS & $1.17(0.94-1.46)$ & $>0.05$ & $1.32(1.04-1.67)$ & $<0.05$ \\
\hline
\end{tabular}

\section{$\mathrm{NC}$ - Not considered}

Table-2: Cox' Stepwise Regression analysis of birth interval components with socio-demographic variables

\begin{tabular}{|c|c|c|c|c|c|c|}
\hline \multirow[t]{2}{*}{ Step } & \multicolumn{3}{|c|}{ PPA } & \multicolumn{3}{|c|}{ Waiting time to conception } \\
\hline & Variable & RR(with 95\%CI) & $\mathrm{P}$-value & Variable & RR(with 95\%CI) & P-value \\
\hline 1 & LACT & $0.98(0.98-0.99)$ & $<0.01$ & LACT & $0.97(0.97-0.98)$ & $<0.01$ \\
\hline \multirow[b]{2}{*}{2} & DPCL & $2.13(1.44-3.14)$ & $<0.01$ & DPCL & $2.63(1.68-4.12)$ & $<0.01$ \\
\hline & LACT & $0.98(0.98-0.99)$ & $<0.01$ & LACT & $0.97(0.96-0.97)$ & $<0.01$ \\
\hline \multirow{3}{*}{3} & DPCL & $2.15(1.46-3.18)$ & $<0.01$ & DPCL & $2.65(1.69-4.15)$ & $<0.01$ \\
\hline & LACT & $0.98(0.98-0.99)$ & $<0.01$ & LACT & $0.97(0.96-0.97)$ & $<0.01$ \\
\hline & FINC & $1.01(1.00-1.01)$ & $<0.01$ & RELH & $0.81(0.70-0.94)$ & $<0.01$ \\
\hline \multirow{4}{*}{4} & DPCL & $2.13(1.44-3.14)$ & $<0.01$ & DPCL & $2.56(1.64-4.02)$ & $<0.01$ \\
\hline & LACT & $0.98(0.98-0.99)$ & $<0.01$ & SPCL & $1.22(1.05-1.41)$ & $<0.01$ \\
\hline & FINC & $1.01(1.00-1.01)$ & $<0.01$ & LACT & $0.97(0.96-0.97)$ & $<0.01$ \\
\hline & RELI & $1.37(1.05-1.79)$ & $<0.05$ & RELH & $0.80(0.69-0.93)$ & $<0.01$ \\
\hline \multirow{5}{*}{5} & DPCL & $2.14(1.45-3.16)$ & $<0.01$ & DPCL & $2.49(1.59-3.91)$ & $<0.01$ \\
\hline & LACT & $0.98(0.98-0.99)$ & $<0.01$ & SPCL & $1.25(1.08-1.45)$ & $<0.01$ \\
\hline & FINC & $1.01(1.00-1.01)$ & $<0.05$ & CDSN & $1.27(1.03-1.57)$ & $<0.05$ \\
\hline & RELI & $1.37(1.05-1.78)$ & $<0.01$ & LACT & $0.97(0.96-0.97)$ & $<0.01$ \\
\hline & TYPF & $0.83(0.69-0.99)$ & $<0.05$ & RELH & $0.81(0.70-0.94)$ & $<0.01$ \\
\hline \multirow{6}{*}{6} & \multirow{6}{*}{\multicolumn{3}{|c|}{ Not found }} & DPCL & $2.51(1.60-3.93)$ & $<0.01$ \\
\hline & & & & SPCL & $1.25(1.08-1.45)$ & $<0.01$ \\
\hline & & & & CDSN & $1.29(1.05-1.58)$ & $<0.05$ \\
\hline & & & & UCDV & $1.17(1.00-1.36)$ & $<0.05$ \\
\hline & & & & LACT & $0.97(0.96-0.97)$ & $<0.01$ \\
\hline & & & & RELH & $0.81(0.70-0.95)$ & $<0.01$ \\
\hline
\end{tabular}

